\title{
Experimental characterization of mechanical properties of the cement paste- aggregate interface in concrete
}

\author{
M. Jebli ${ }^{1,3, \star}$, F. Jamin ${ }^{1,3}$, E. Malachanne ${ }^{2}$, E. Garcia-Diaz ${ }^{2}$, and M.S. El Youssoufi ${ }^{1,3}$ \\ ${ }^{1}$ LMGC, Univ. Montpellier, CNRS, Montpellier, France \\ ${ }^{2} \mathrm{C} 2 \mathrm{MA}$, Ecole des mines d'Alès, Alès, France \\ ${ }^{3}$ MIST Lab., IRSN, CNRS, Univ. Montpellier, France
}

\begin{abstract}
Granular materials are extensively used in the field of civil engineering. These materials are either used in their dry state, or mixed with water or with a binder. In the case of concrete, the binder could be cement or mortar. For ordinary concretes, it is generally admitted that there is a thin heterogeneous zone of paste, with a thickness of about $15-60 \mu \mathrm{m}$, surrounding the aggregates surface. This zone, commonly named the Interfacial Transition Zone (ITZ), is characterized by a higher porosity than the bulk paste and a high concentration of the portlandite crystals. Some of these crystals react with the aggregates' surface (limestone aggregates), leading to a good adhesion. In this work, the mechanical properties of the cement paste and of the cement-aggregate interface are experimentally analyzed. Experimental tensile and shear tests are performed on parallelipipedic samples. These samples are made by linking limestone aggregates with Portland cement paste using a water / cement ratio of 0.5 . The results show that the cement-aggregate interface is the weak zone in the composite.
\end{abstract}

\section{Introduction}

Concrete is a composite material of aggregate particles and cement paste. The mechanical properties of concrete depend on the properties of the cement paste, the aggregates and the cement paste-aggregate interface. The cementaggregate interface is characterized by a higher porosity than that found in the bulk paste. For this reason, the mechanical properties of this zone are considered different than those of the cement paste.

There is ample evidence that the properties of concrete, such as the tensile strength, modes of failure and the permeability; are significantly influenced by the nature of the existing interfaces between the cement paste and the aggregates. However, due to lack of understanding about these interfaces, very little attempts have been made to characterize the mechanical properties of this zone. Some studies have been devoted to the direct characterization of the mechanical properties of the interface between aggregates and cement paste, by using tensile and shear tests.

The literature of bond strength prior to 1965 has been admirably reviewed by Alexander et al. [1] and by Shah and Slate [2]. Both papers support the finding that the tensile strength of the cement-aggregate bond is lower than that of cement paste. Recently, Rao and Prasad [3] and Gu et al. [4] found that the tensile strength of the interface between aggregate and mortar was about one third to one half of the tensile strength of the mortar. Mielniczuk et al. [5] and Jebli et al. [6] have characterized the behavior of a composite with spherical aggregate using compression

\footnotetext{
^e-mail: mouad.jebli@umontpellier.fr
}

and tensile tests. They showed, at this scale, that the proposed models on the macroscopic scale by Eurocode 2 are confirmed to predict behavior at rupture.

In conclusion, all experimental studies to characterize the interface between cement paste and aggregate remain insufficient to assess the behavior law at local scale. In this context, the objective of this study is to determine the mechanical properties of the interface between the aggregate and the cement paste composite by direct tensile and shear tests.

\section{Experimental program}

The selected cement is a Portland cement CEM II 32.5 $\mathrm{CP} 2 \mathrm{NF}$ used to prepare all composites with a water/cement ratio of 0.5 .

\subsection{Preparation for samples}

Natural limestone parallelepipedic aggregates were made with dimensions of $10 \times 10 \times 15 \mathrm{~mm}^{3}$.

In this experimental study, two types of samples whose dimensions are shown in Figure 1, were made:

- For tensile tests, the samples have a thin cement paste layer of $2 \mathrm{~mm}$ between two limestone aggregates (Fig. 1a).

- For direct shear tests, the samples are composed of a limestone aggregate and cement paste (Fig.1b).

The samples were prepared using specific molds adapted to the desired dimensions of the composite. All samples 
a)

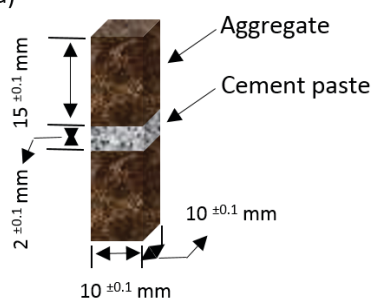

b)

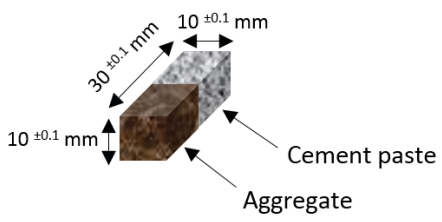

Figure 1. Sample sizes for direct tensile test a) and shear test b)

are demolded after 24 hours of hydration and are immediately immersed in water saturated with lime at a temperature of $21^{\circ} \mathrm{C}$ to prevent the leaching and to decrease concrete shrinkage.

At different times of hydration (2, 7, 15, 28 and 90 days), we examine both the direct tensile and the shear bond strengths of the composite by means of specific devices. The shear tests are conducted for different normal stresses. All the tests are designed to characterize mechanical interaction laws at the local scale of the cement paste-aggregate interface.

To obtain the mechanical properties of the cement paste, mechanical tests were also carried out on cement paste samples of dimensions equal to those of composites.

\subsection{Direct tensile test}

To perform mechanical tests of direct tensile, experimental devices were designed and manufactured to be adapted to the size and shape of the desired samples. The principle of these tests is simple, but the preparation and implementation should be made carefully. Indeed, each mis-alignment of the supports or in the tilt sample induced parasitic bending which changes the results. Four metal shims with holes are first fixed onto the sample as shown in Figure 2. To perform the test, the sample is fixed by placing the metal rods in the holes of shims and of the U-shaped support set on the machine.
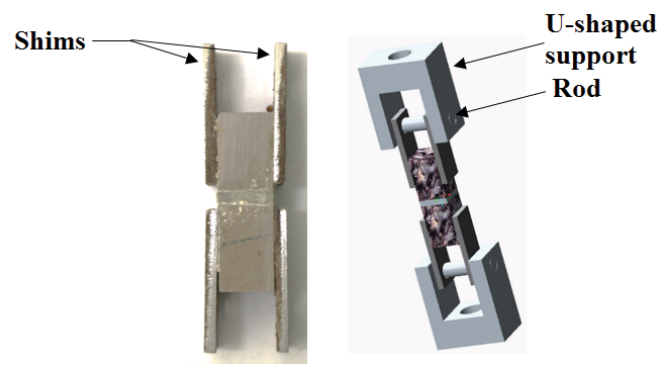

Figure 2. Experimental device for direct tensile test

\subsection{Direct shear test}

This test is among the most difficult tests to be performed on cementitious materials, especially to characterize the cement paste-aggregate interface due to the lack of appropriate experimental tools. The objective of our original approach is to access the Mohr-Coulomb law of cement paste-aggregate interface (local cohesion and friction angle). To perform this test, experimental devices were designed and manufactured in a way that the rupture is located at the cement paste-aggregate interface. The device consists of two half-boxes, the distance between these two half-boxes is around $0.5 \mathrm{~mm}$ to minimize the sample bending. The interface is fixed in the middle of the device with screws. These crews are also used to apply a normal force. Figure 3 illustrates the experimental apparatus in which the sample is placed inside the two half-boxes.

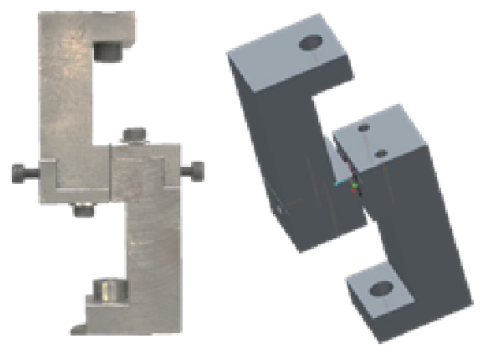

Figure 3. Experimental device for direct shear test

All tests were performed on a "Mechanical Testing System" by imposing a constant loading speed of 0.01 $\mathrm{mm} / \mathrm{s}$. Mechanical tests were filmed with a fast camera Vision Research Phantom V12 (acquisition speed of 10 to 40 thousand pictures per second), to correct the displacement during the tests by image correlation.

\section{Experimental results}

It is proposed to present the experimental results obtained from the direct tensile and shear tests, on cement paste and the composite. For all the tensile and shear tests, it is observed that all the breaks on the composite occur at the cement-aggregate interface (Fig. 4), which represents the weakest zone.

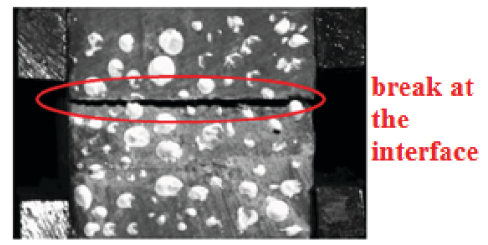

Figure 4. Crack growth at interface during tensile test

\subsection{Direct tensile tests}

Figure 5 shows the stress evolution as a function of the strain respectively for the composite "cement pasteaggregate" (Fig. 5a) and for pure cement paste (Fig. 5b). Significant increase in strength $\sigma_{r}(j)$ at the beginning of hydration, and a progressive stabilization after three weeks 
of hydration for the composite and cement paste is observed.
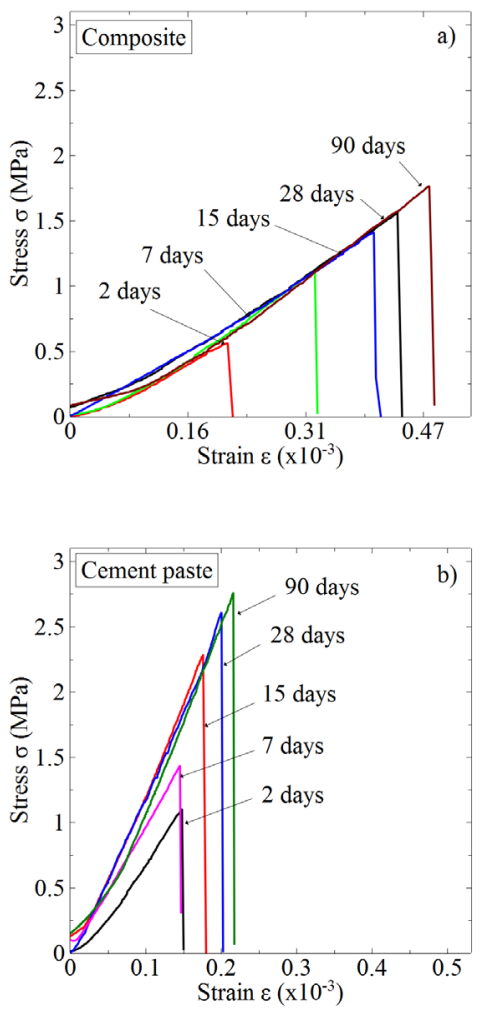

Figure 5. Evolution of the stress vs strain during tensile tests for composite a) and cement paste b)

Figure 6 shows the evolution of the strength $\sigma_{r}(j)$ in function of hydration time $j$ during tensile tests for cement paste and composite. The results show a similar evolution of the strength, but at each hydration time the cement paste has higher characteristics than the composite. In addition, ratio between composite and cement paste is about $2 / 3$ for the tensile strength. These results tend to confirm that the cement paste-aggregate interface is the weakest zone in composite. An increase of the strain values is also observed with curing, with higher values for the composite than that for the cement paste. Indeed this shows the more "ductile" character of the composite than of the cement paste.

\subsection{Direct shear tests}

The curves of stress - strain, obtained during direct shear tests (without normal stress), are presented in Figure 7 at different hydration times during the first 40 days for the cement paste and composite. These figures show a fast increase in the shear strength and stiffness of the samples at the beginning of hydration. We noticed a decrease in the evolution speed of the shear strength after 28 days. With both the shear test and the tensile test, a rapid drop in stress is observed just after it reached its peak. So, an absence of the softening part of the stress-strain curve is observed in macroscopic conventional mechanical behavior under shear stress. An increase of the strain values is

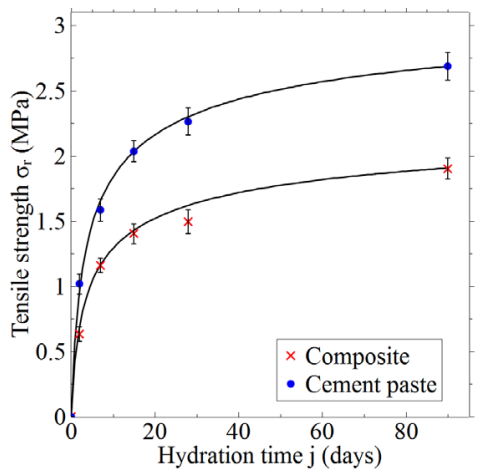

Figure 6. Evolution of tensile strength as a function of hydration time during tensile tests for composite and cement paste

also observed in the case of the composite with curing, with lightly higher values of the composite beyond 7 days than that of the cement paste. In this case the crack spreads quickly in the cement-aggregate interface. For the cement paste, breaking is conducted in the same strain value whatever the time of hydration.
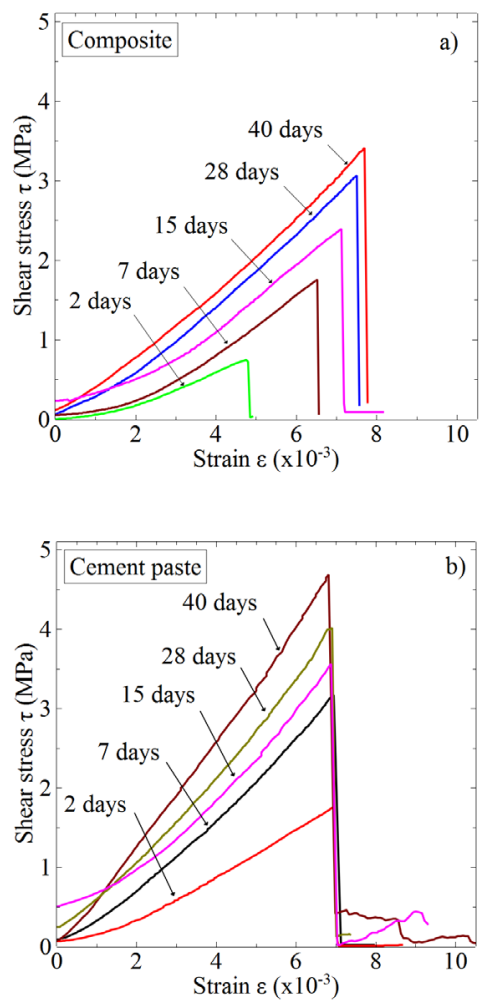

Figure 7. Shear stress-strain curves for composite a) and cement paste b)

The values of strength $\tau_{r}(j)(\mathrm{MPa})$ as a function of the hydration time $j$ (in days), for the shear test for the cement paste and the composite, are shown on Figure 8. This figure shows a similar increase in strength during hydration time for both types of samples. After the characterization of the effect of hydration time on the shear strength, respectively for pure cement paste and for the cement pasteaggregate interface, we focus on the influence of normal 


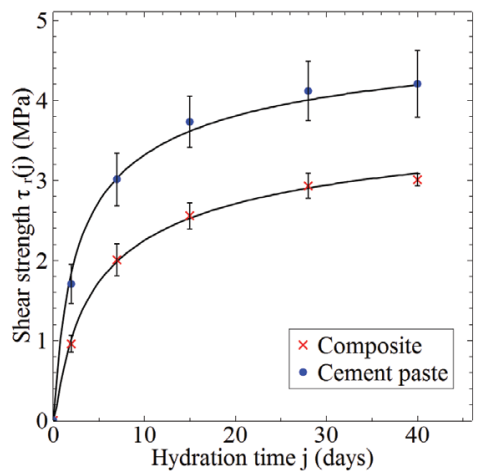

Figure 8. Evolution of the shear strength as a function of hydration time for the composite and the cement paste

stress on the shear strengths. The samples, at a fixed hydration time (40 days), are subjected to direct shear tests at different normal stresses. The obtained stress-strain curves are shown in Figure 9 for different normal stresses $(0,0.5$, $1,1.5 \mathrm{MPa}$ ).
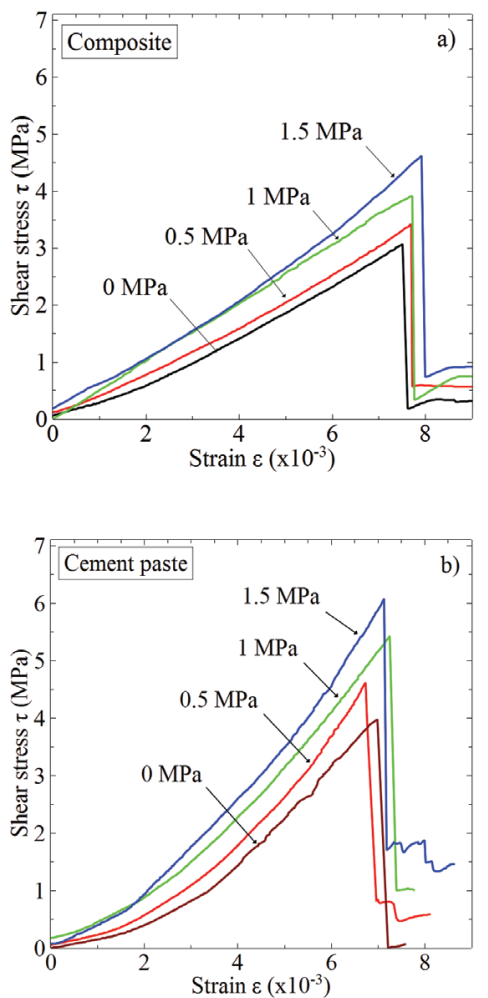

Figure 9. stress-strain curves during shear tests for composite a) and cement paste b) at different normal stresses and at 40 days

We noticed that there is a strong dependence of mechanical behavior of composite and cement paste on normal stress. For both samples, an increase in shear strength due to application of normal stress is noted. The initial tangential modulus also increases in the same way. This evolution of shear strength according to normal stress (Fig. 10) is used to calculate the local friction angle and cohesion according to the Mohr-Coulomb criterion. We noticed a linear evolution of the shear strength with the increase of normal stress, for both cement paste and composite. The results, presented in Table 1, show that the local cohesion $c$ and the local friction angle $\Phi$ of the paste are greater than that of the composite.

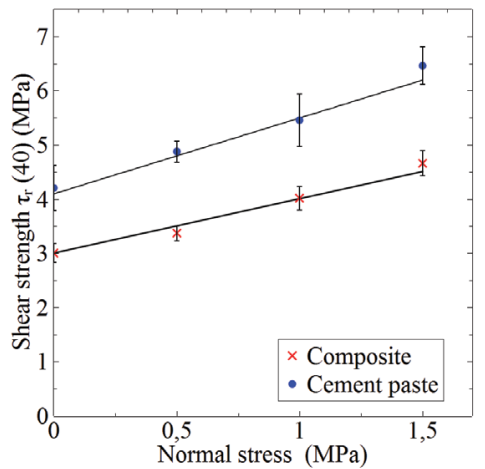

Figure 10. Mohr - Coulomb criterion for the composite and the cement paste at 40 days of hydration

Table 1. Local cohesion $c$ and friction angle $\Phi$ of the composite and the cement paste at 40 days of hydration

\begin{tabular}{|c|c|c|}
\hline & Cement paste & Composite \\
\hline Local cohesion $c(\mathrm{MPa})$ & 4.1 & 2.9 \\
\hline Local friction angle $\Phi\left(^{\circ}\right)$ & 54 & 47 \\
\hline
\end{tabular}

\section{Conclusion}

These results are a first approach to characterize the mechanical properties of the interface with the original experimental tools. The experimental results showed that the cement-aggregate interface is the weakest zone in composite. For both tests the strain values of the composite are higher than those of cement paste. Indeed, the more "ductile character" of the composite compared to the cement paste was more marked for the tensile test than for the shear test. These results at the local scale will improve numerical models (eg CZM) by identifying the parameters to be included in the calculation codes.

\section{References}

[1] K.M. Alexander, D.J. Gilbert, J. Wardlaw (1968)

[2] S.P. Shah, F.O. Slate, Cement and Concrete Assoc, London UK (1968)

[3] G.A. Rao, B.R. Prasad, Cement and Concrete Research 32, 253 (2002)

[4] X. Gu, L. Hong, Z. Wang, F. Lin, Construction and Building Materials 46, 156 (2013)

[5] B. Mielniczuk, M. Jebli, F. Jamin, M.S. El Youssoufi, C. Pelissou, Y. Monerie, Cement and Concrete Research 79, 235 (2016)

[6] M. Jebli, F. Jamin, E. Garcia-Diaz, M. El Omari, M.S. El Youssoufi, Cement and Concrete Composites 12, 241 (2016) 